\title{
Dipole-strength functions below the giant dipole resonance in the stable even-mass molybdenum isotopes
}

G. Rusev ${ }^{* a}$, R. Schwengner ${ }^{a}$, R. Beyer ${ }^{a}$, F. Dönau ${ }^{a}$, M. Erhard ${ }^{a}$, E. Grosse ${ }^{a, b}$, A. R. Junghans ${ }^{a}$, K. Kosev ${ }^{a}$, J. Klug ${ }^{a}$, C. Nair ${ }^{a}$, N. Nankov ${ }^{a, c}$, K. D. Schilling ${ }^{a}$ and A. Wagner ${ }^{a}$

${ }^{a}$ Institut für Strahlenphysik, Forschungszentrum Dresden-Rossendorf, 01314 Dresden, Germany

${ }^{b}$ Institut für Kern- und Teilchenphysik, Technische Universität Dresden, 01062 Dresden, Germany

${ }^{c}$ Institute for Nuclear Research and Nuclear Energy, BAS, 1784 Sofia, Bulgaria

E-mail: rusev@tunl.duke.edu

\begin{abstract}
Dipole-strength distributions in the stable even-mass molybdenum isotopes up to the neutronseparation energies have been studied in photon-scattering experiments with bremsstrahlung at the superconducting electron accelerator ELBE of the Research Centre Dresden-Rossendorf. In order to determine the dipole-strength distribution up to the neutron emission threshold statistical methods were developed for the analysis of the measured spectra. The measured spectra of scattered photons were corrected for detector response and atomic background by simulations using the code GEANT3. Simulations of $\gamma$-ray cascades were performed in order to correct the intensities of ground-state transitions for feeding from high-lying levels and to determine branching ratios. The photoabsorption cross sections obtained for the stable even-mass molybdenum isotopes from the present $(\gamma, \gamma)$ experiments are combined with $(\gamma, n)$ data from literature resulting in a photoabsorption cross section covering the full range from about 4 to about $15 \mathrm{MeV}$, which is of interest for nuclear astrophysics network calculations. Novel information about the low-energy tail of the Giant Dipole Resonance and the energy spreading of its strength is derived. The dipole-strength distributions deduced from the present photon-scattering experiments show that the dipole strength increases with the neutron number of the Mo isotopes. The experimental results are discussed in the frame of Quasiparticle-Random-Phase-Approximation calculations in a deformed basis which describe the increasing strength as a result of the increasing deformation.
\end{abstract}

Workshop on Photon Strength Functions and Related Topics

June 17-20 2007

Prague, Czech Republic

\footnotetext{
*Speaker.

†Present address: TUNL, Duke University, Durham, North Carolina 27708, USA
} 


\section{Introduction}

Among the collective modes of nuclei the electric dipole $(E 1)$ excitation has the special property that most of its strength is concentrated in the isovector Giant Dipole Resonance (GDR). Macroscopically this strong resonance is described as a vibration of the charged (proton) matter in the nucleus against the neutral matter (neutrons) [1, 2, 3]. It is a long standing question of nuclear physics to specify how much of the $E 1$ strength is still present at energies far below the GDR maximum. Theoretically it has been shown [4] that it is justified to describe the GDR by a Lorentzian also below the particle emission thresholds. Here it is important that the contribution of particle emission, i.e. the escape widths, to the total width of the GDR is negligible [5]. Various experimental attempts to clarify this question for heavier nuclei have led to conflicting results. Neutron capture experiments often have indicated an overshoot of the Lorentzian over the observed $E 1$ strength at the low energy tail of the GDR [6]. On the basis of these data theoretical explanations have been proposed [7] to explain the differences which include the posit of a strong energy dependence of the GDR width. Photon-scattering experiments on the other hand are in some cases in reasonable agreement with the Lorentzian extrapolation $[8,9]$. Since even extra strength was identified [10] the extra identifier "pygmy resonance" was invented [11, 12]. As in the last years significant progress has been made in the field of photon-induced nuclear excitations new experimental attempts to clarify the situation are well justified.

The measurement of the photoabsorption cross section $\sigma_{\gamma}$ at energies close to the neutron separation energy is faced with the following problems: Below the neutron threshold $\sigma_{\gamma}$ can be measured via $\gamma$ rays emitted after photoexcitation. However, the increasing density of nuclear states towards the threshold leads to complex deexcitation patterns that include not only the deexcitation to the ground state but also to many intermediate states. Hence, the intensities of the ground-state transitions drop rapidly towards high excitation energies while many branching transitions to intermediate states appear in the low- and medium-energy part of the $\gamma$-ray spectrum. Because the assignment of the several hundred transitions observed in a spectrum to certain states is practically impossible, the determination of the dipole-strength distribution at high energies requires an appropriate method to correct for their impact on the data. Above the $(\gamma, n)$ threshold $\sigma_{\gamma}$ has been measured by detecting neutrons following photoabsorption [13]. Apparently, the absorption cross section closely above the threshold for neutron emission is small and accurate data are difficult to obtain for these energies [14]. The theoretical prediction of the distribution of the dipole strength around the neutron threshold is also a highly challenging task. A fair description of the qualitative features of the GDR has been achieved by empirical and microscopic models. However, the quantitative calculation of the width, in particular the spreading part, is missing such that extrapolations of the GDR distributions towards lower energies are not reliable. Summarising we feel the lack of both nuclear data and nuclear theory impact for a better prediction of the photo cross section in the critical threshold region.

In this paper we present results of photon-scattering experiments in the energy region from 4 $\mathrm{MeV}$ to the neutron emission thresholds for the sequence of stable even-mass molybdenum isotopes with mass numbers from $A=92$ to 100, performed at the superconducting electron accelerator ELBE of the Forschungszentrum Dresden-Rossendorf. For the first time photon-scattering spectra are corrected with respect to branching and feeding transitions by applying statistical methods. In 
this way, we obtain information about the behaviour of the photoabsorption cross section in the region below the neutron-separation energy and, thus, about the low-energy tail of the GDR. We compare the experimental findings with predictions of a Quasiparticle-Random-Phase-Approximation (QRPA) in a deformed basis and show the influence of the nuclear shape on the behaviour of the photoabsorption cross section.

\section{Experimental Considerations}

\subsection{Photon scattering}

In photon scattering, a level in a stable nucleus is populated by the resonant absorption of a real photon. The level can deexcite to the ground state or to some intermediate levels by $\gamma$-ray transitions. The process of photon scattering is called Nuclear-Resonance-Fluorescence (NRF) $[15,16]$. The probability to excite a level with spin $J_{R}$ from the ground-state with spin $J_{0}$ is given by the photoabsorption cross section integral $I_{R}$ over the resonance $R$ :

$$
I_{R}=\int_{0}^{\infty} \sigma_{\gamma}(E) d E=\frac{2 J_{R}+1}{2 J_{0}+1}\left(\frac{\pi \hbar c}{E_{R}}\right)^{2} \Gamma_{0},
$$

where $E_{R}$ is the energy of the populated level with angular momentum $J_{R}$ and $\Gamma_{0}$ is the partial width of the ground-state transition. Because of the low momentum transfer in photon-scattering mainly levels with spin $J_{R}$ satisfying the condition $\left|J_{0}-1\right| \leq J_{R} \leq J_{0}+1$ are excited.

In the case of non-overlapping resonances, NRF is described to proceed via a compoundnucleus reaction with uncorrelated channels $f$ characterised by the partial widths $\Gamma_{f}$. The photonscattering cross section $\sigma_{\gamma f}$ representing the process of excitation of a level $E_{R}$ and deexcitation to a level $E_{f}$ is expressed as:

$$
\sigma_{\gamma f}\left(E_{R}\right)=\sigma_{\gamma}\left(E_{R}\right) \frac{\Gamma_{f}}{\Gamma}
$$

where all partial widths contribute to the total level width $\Gamma=\sum \Gamma_{f}$. In integral form, Eq. (2.2) can be written as:

$$
I_{s}=\int_{0}^{\infty} \sigma_{\gamma f}(E) d E=\frac{2 J_{R}+1}{2 J_{0}+1}\left(\frac{\pi \hbar c}{E_{R}}\right)^{2} \Gamma_{0} \frac{\Gamma_{f}}{\Gamma}
$$

where $I_{s}$ is the scattering cross section integral for the level $R$ and $\Gamma_{f}$ is the partial width for a transition from $R$ to a level $f$. The case of $f=0$ corresponds to elastic scattering, i.e. the deexcitation transition is the one to the ground state. The corresponding elastic scattering cross section is labelled $\sigma_{\gamma \gamma}$. The determination of $\Gamma_{0}$ from $I_{s}$ for a level $R$ excited by absorption of a photon from the ground state requires the identification of all transitions deexciting the level $R$ in order to calculate the branching ratios $\Gamma_{f} / \Gamma$ (cf. Eq. (2.3)). The intensity $I_{\gamma}\left(E_{\gamma}, \theta\right)$ of a considered ground-state transition at $E_{\gamma}=E_{R}$ measured at an angle $\theta$ relative to the incident photon beam is expressed as:

$$
I_{\gamma}\left(E_{\gamma}, \theta\right)=I_{s}\left(E_{R}\right) \Phi\left(E_{R}\right) \varepsilon\left(E_{\gamma}\right) N_{\mathrm{at}} W(\theta) \frac{\Delta \Omega}{4 \pi},
$$

where $W(\theta)$ is the angular correlation function of this transition, $\Delta \Omega$ in the solid angle under which the detector views the sample, $\Phi\left(E_{R}\right)$ is the absolute photon flux at $E_{R}, \varepsilon\left(E_{\gamma}\right)$ the absolute full-energy-peak efficiency and $N_{\text {at }}$ the number of atoms in the sample. 
In our photon-scattering experiments we used bremsstrahlung produced by deceleration of electrons in a thin metallic foil (radiator) which allows an excitation of many levels in a wide energy range. If the electron energy is high enough above a particular level, the NRF experiments with bremsstrahlung lead to the possibility of the population of a level by a branching transition from a higher lying level. Such feeding increases the intensity of the ground-state transition of the considered resonance $R$. The intensity of the ground-state transition becomes a superposition of the rate of elastic scattering and the intensity of the feeding transitions to $R$. The cross section integral $I_{s+f}$ deduced for this case can be expressed in a more general form as:

$$
\begin{array}{r}
I_{s+f}=\int_{0}^{\infty} \sigma_{\gamma \gamma}(E) d E+\sum_{i>R} \int_{0}^{\infty} \sigma_{\gamma i}(E) \frac{\Gamma_{0}}{\Gamma} d E= \\
\frac{2 J_{R}+1}{2 J_{0}+1}\left(\frac{\pi \hbar c}{E_{R}}\right)^{2} \frac{\Gamma_{0}^{2}}{\Gamma}+\sum_{i>R} \frac{\Phi\left(E_{i}\right)}{\Phi\left(E_{R}\right)} \frac{2 J_{i}+1}{2 J_{0}+1}\left(\frac{\pi \hbar c}{E_{i}}\right)^{2} \Gamma_{0}^{i} \frac{\Gamma_{R}^{i}}{\Gamma^{i}} \frac{\Gamma_{0}}{\Gamma},
\end{array}
$$

where summation over $i>R$ means that the energy $E_{i}$ of a level which feeds the considered resonance $R$ is higher than energy $E_{R}$ of this resonance. Quantities $\Gamma^{i}, \Gamma_{0}^{i}$ and $\Gamma_{R}^{i}$ are the total width of the level $E_{i}$, its ground-state width and the partial decay width to the level $R$, respectively. Taking into account the elastic and inelastic photon scattering, the intensity of the ground-state transition deexciting the resonance $R$ can be obtained from Eq. (2.4) by substitution of $I_{s}$ with the expression given in Eq. (2.5).

It is evident that the correct determination of the scattering integral from photon-scattering experiments with bremsstrahlung requires a correction for the intensity of the feeding transitions and for the branching ratio $\Gamma_{0} / \Gamma$.

The radiative strength functions characterise the excitation and deexcitation $\gamma$-ray transitions in the nucleus. The strength function is defined as average reduced transition width, see, e.g., Ref. [11]. In general, two types of strength function are distinguished: (i) the "downward" strength function $\bar{f}_{X L}\left(E_{\gamma}\right)$ related to the deexcitation transitions:

$$
\overleftarrow{f}_{X L}\left(E_{\gamma}\right)=E_{\gamma}^{-(2 L+1)} \frac{\left\langle\Gamma_{i}^{X L}\left(E_{\gamma}\right)\right\rangle}{D}
$$

and (ii) the "upward" strength function $\vec{f}_{X L}\left(E_{\gamma}\right)$ related to the excitation of the levels from the ground state, i.e. depending only on the $\Gamma_{0}$ widths:

$$
\vec{f}_{X L}\left(E_{\gamma}\right)=E_{\gamma}^{-(2 L+1)} \frac{\left\langle\Gamma_{0}^{X L}\left(E_{\gamma}\right)\right\rangle}{D},
$$

where $\Gamma_{i}^{X L}\left(E_{\gamma}\right)$ is the partial width for a transition to level $i$ (i=0 is the ground state) of type $X$, multipolarity $L$ and energy $E_{\gamma} . D$ is the average spacing of the levels near the excited resonance $E_{R}$. In the case of photon scattering, radiation with multipolarity $L>1$ contributes only weakly to the absorption of photons, such that $\vec{f}_{X L}\left(E_{\gamma}\right)=\vec{f}_{M 1}\left(E_{\gamma}\right)+\vec{f}_{E 1}\left(E_{\gamma}\right)+E_{\gamma}^{2} \vec{f}_{M 2}\left(E_{\gamma}\right)+E_{\gamma}^{2} \vec{f}_{E 2}$ $\left(E_{\gamma}\right)+\ldots \approx \vec{f}_{1}\left(E_{\gamma}\right)$. According to Eq. (2.1), the "upward" dipole-strength function can be related to the average photoabsorption cross section $\left\langle\sigma_{\gamma}\right\rangle$ :

$$
\vec{f}_{1}\left(E_{\gamma}\right)=\frac{2 J_{0}+1}{2 J_{R}+1} \frac{\left\langle\sigma_{\gamma}\left(E_{R}\right)\right\rangle}{(\pi \hbar c)^{2} E_{R}} .
$$

Note that for the excitation $E_{R}=E_{\gamma}$. 


\subsection{Simulations of $\gamma$-ray cascades}

In photon-scattering experiments, a fraction of the observed $\gamma$-rays results from ground-state transitions. The ratio of the intensities of the ground-state transitions to that of branching transitions decreases with increasing excitation energy because of the large number of intermediate levels available from the deexcitation of the initial one. The determination of the branching transitions becomes increasingly difficult at high $(>4 \mathrm{MeV})$ energy. Therefore, we apply statistical methods to estimate the intensities of the branching transitions relative to the ground-state transitions.

We developed a Monte Carlo code for the simulation of $\gamma$-ray cascades analogously to the strategy of the code DICEBOX [17]. The nucleus is modelled by a nuclear realisation consisting of (i) a level scheme including levels with spin $J=0,1$, and 2 and (ii) an assignment of partial decay widths for $E 1, M 1$ or $E 2$ transition depopulating every level.

The level density is calculated according to the back-shifted Fermi gas model. The leveldensity parameter $a$ and the back-shift energy $E_{1}$ are taken from the systematics presented in Ref. [18]. The densities of levels with positive parity and with negative parity are assumed to be equal according to Ref. [19]. The Wigner distribution (see, e.g., Ref. [20]) is used for the fluctuation of the nearest-neighbour spacings of levels with the same spin in order to include the effect of level repulsion. Optionally in the program, the level density can be calculated in the constant temperature approximation.

A priori strength functions for $E 1, M 1$ and $E 2$ transitions are used to calculate the average decay widths of the levels. The strength function for the $E 1$ transitions is derived form a parametrisation of the Giant Dipole Resonance for triaxialy deformed nuclei presented in the contribution by E. Grosse to these proceedings. The strength function for $M 1$ transitions is taken from QRPA calculations in a deformed basis for the stable even-mass Mo isotopes [21]. The QRPA calculations reproduce the scissors mode and the spin-flip $M 1$ resonance in the Mo isotopes but with strength smaller than the measured one. We normalise the calculated $M 1$ strength to the measured one in the excitation range up to $4 \mathrm{MeV}$. It was shown from data from two-step cascades following the thermal neutron capture in ${ }^{162} \mathrm{Dy}[22,23]$ that the scissors mode and the $M 1$ spin-flip resonance are built not only in the ground state but also on excited states including the levels in the quasicontinuum in accordance with the Brink hypothesis. The strength function for $E 2$ transitions is taken from a global parametrisation of the E2 Isoscalar Giant Resonance provided by RIPL [24]. The Porter-Thomas distribution [25] is used for the fluctuations of the partial decay widths of the levels around the average obtained from the strength function.

The simulation of the $\gamma$-ray cascades starts with the excitation of a level with spin $J=1$ according to the photoabsorption cross section (cf. Eq. (2.1)). The deexcitation of the level is governed by the branching ratios for decay to the ground state or to any intermediate level calculated from the partial decay widths $B_{f}=\Gamma_{f} / \Gamma$. If the decay is not to the ground state then the populated intermediate level is considered as an excited level and a new transition is performed until the ground state is reached. The code provides the energy, the spin and parity of the initial and the final level as well as the multipole order of the transition. Since one nuclear realisation does not represent completely the properties of the nucleus, but is only a sample, the cascade simulations have to run for many nuclear realisations. The simulations presented in this paper were performed for 1000 nuclear realisations. 


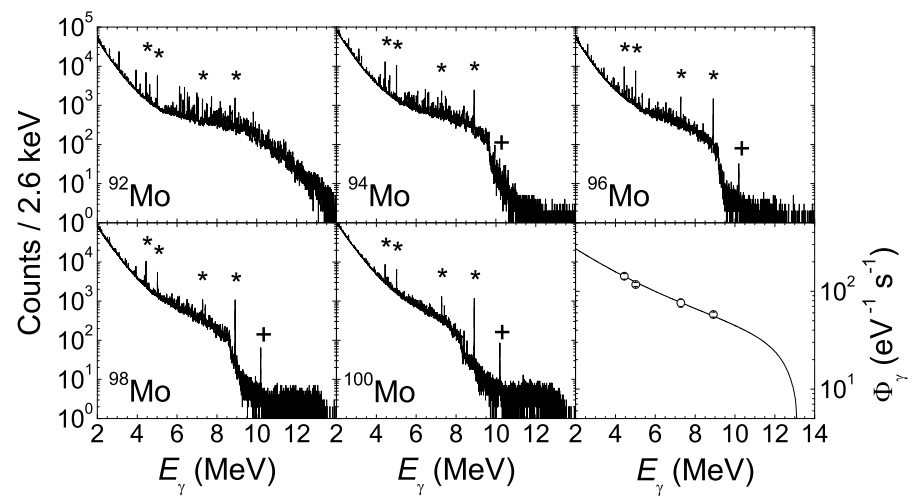

Figure 1: Spectra of photons scattered from ${ }^{92} \mathrm{Mo},{ }^{94} \mathrm{Mo},{ }^{96} \mathrm{Mo},{ }^{98} \mathrm{Mo}$ and ${ }^{100} \mathrm{Mo}$ at $127^{\circ}$ relative to the incident beam. The measurements were performed at identical experimental conditions and an electronbeam energy of $13.2 \mathrm{MeV}$. Strongest peaks labelled with * are from the calibration standard ${ }^{11} \mathrm{~B}$. The peaks labelled with + are from $(n, \gamma)$ reaction on ${ }^{73} \mathrm{Ge}$. The spectrum of the incident photon beam is shown on the bottom right figure. It is normalised to the flux deduced from transitions in ${ }^{11} \mathrm{~B}$.

\section{Experimental Results}

The present paper reports on photon-scattering experiments for the stable even-mass molybdenum isotopes ${ }^{92} \mathrm{Mo},{ }^{94} \mathrm{Mo},{ }^{96} \mathrm{Mo},{ }^{98} \mathrm{Mo}$ and ${ }^{100} \mathrm{Mo}$ carried out at the bremsstrahlung facility at the superconducting electron accelerator ELBE of the Forschungszentrum Dresden-Rossendorf. Bremsstrahlung was produced by electrons hitting a $7 \mu \mathrm{m}\left(3.4 \mathrm{mg} / \mathrm{cm}^{2}\right)$ thick Nb radiator. A narrow photon beam is formed by an Al collimator with a length of $2.6 \mathrm{~m}$ and an opening angle of $5 \mathrm{mrad}$. An absorber made of a $10 \mathrm{~cm}$ long $\mathrm{Al}$ cylinder between the radiator and the collimator attenuates the intense low energy part of the bremsstrahlung spectrum. The photon beam irradiates the target shaped as a disk with a diameter of $2 \mathrm{~cm}$. For background reduction, the target was placed in an evacuated polyethylene tube and the photon beam was absorbed in a well shielded photon-beam dump after passing the target. The photons scattered from the target were registered in four high-purity Ge detectors (HPGe) with an efficiency of $100 \%$ relative to a 3 in. $\times 3$ in. NaI detector. In order to determine the multipole order of the scattered $\gamma$ rays two of the detectors were located at $127^{\circ}$ and the other two at $90^{\circ}$ with respect to the photon beam at distances of 32 and $28 \mathrm{~cm}$ to the target, respectively. The low-energy photons are suppressed by lead absorbers with a thickness of 0.8 and $1.3 \mathrm{~cm}$ at the two given angles, respectively, combined with $0.3 \mathrm{~cm}$ thick copper absorbers. The HPGe detectors are equipped with $3 \mathrm{~cm}$ thick escape-suppression shields made of BGO scintillation detectors. The detector resolution is $5 \mathrm{keV}$ and $8 \mathrm{keV}$ at $5 \mathrm{MeV}$ and 9 $\mathrm{MeV}$, respectively. The bremsstrahlung facility of the ELBE accelerator is described in detail in Refs. [26, 27].

We performed five measurements on the stable even-mass molybdenum isotopes at identical experimental conditions and the same electron-beam energy $E_{e}^{\mathrm{kin}}=13.2 \mathrm{MeV}$. Samples of elementary ${ }^{92} \mathrm{Mo},{ }^{94} \mathrm{Mo},{ }^{96} \mathrm{Mo},{ }^{98} \mathrm{Mo}$ and ${ }^{100} \mathrm{Mo}$ isotopically enriched to $97.31 \%, 98.97 \%, 95.90 \%$, $98.55 \%$ and $99.27 \%$ with masses of 2036, 1998, 2003, $2953 \mathrm{mg}$ and $2917 \mathrm{mg}$, respectively, were used as targets. Spectra of photons scattered at $127^{\circ}$ from the molybdenum isotopes are presented 


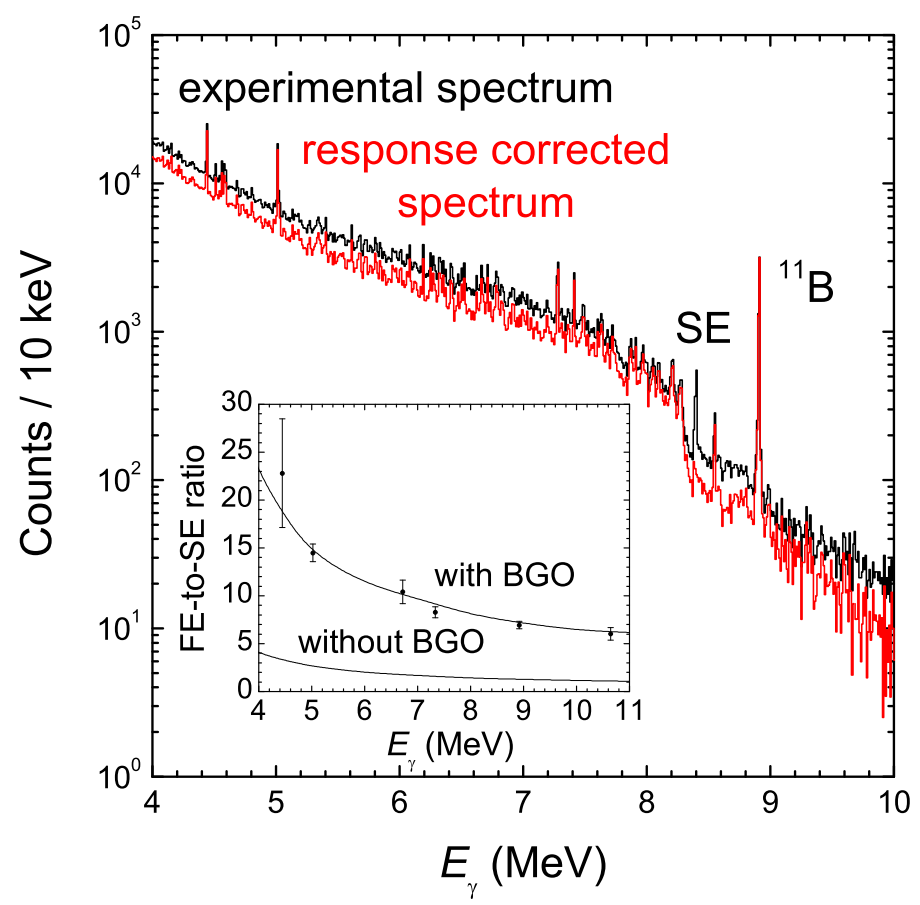

Figure 2: Raw spectrum of photons scattered from ${ }^{100} \mathrm{Mo}$ at $127^{\circ}$ in the experiment at $E_{e}^{\mathrm{kin}}=13.2 \mathrm{MeV}$ (black) compared with the result of the correction accounting for the detector response (red). A comparison of the measured ratio of the full-energy peak to the single-escape peak (symbols) with results from GEANT3 simulations (solid line) is shown in the insert.

in Fig. 1. Photon flux in the ${ }^{100}$ Mo experiment deduced from the transitions in ${ }^{11} \mathrm{~B}$ is compared with the approximation for the bremsstrahlung spectrum given by Roche et al. [28, 29] in Fig. 1.

Simulations for the detector response were performed using the program code GEANT3 [30]. A comparison of the raw spectrum of ${ }^{100} \mathrm{Mo}$ and the spectrum after detector-response correction is presented in Fig. 2. A comparison of the results for the ratio of the full-energy peak to the single-escape peak (FE-to-SE ratio) obtained from the simulations and measurements of the photon-scattering from ${ }^{11} \mathrm{~B},{ }^{16} \mathrm{O}$, and ${ }^{28} \mathrm{Si}$ is shown in the insert in Fig. 2. The detector response simulations are used for estimate of the full-energy peak efficiency, see Fig. 2 in Ref. [31].

\subsection{Determination of non-nuclear background radiation}

The high level density and the Porter-Thomas fluctuations of the decay widths cause many weak transitions which can not be observed as isolated peaks. In addition, every level can decay to many intermediate levels via weak transitions. Therefore not all the intensity is carried by observed peaks. Many of the transitions are so weak that their superposition is observed as a quasicontinuum. An estimate for the intensity of resonant scattering "hidden" in the background can be obtained from a comparison of spectra of $\gamma$-rays scattered from different isotopes. The spectra measured at $127^{\circ}$ in the photon-scattering experiments on ${ }^{98} \mathrm{Mo}$ and ${ }^{100} \mathrm{Mo}$ at $E_{e}^{\mathrm{kin}}=13.2 \mathrm{MeV}$ are presented in Fig. 3 (a). The spectra are corrected for the natural background, and for the detector response. Additional the spectra are corrected for the full-energy peak efficiency, photon flux, area 


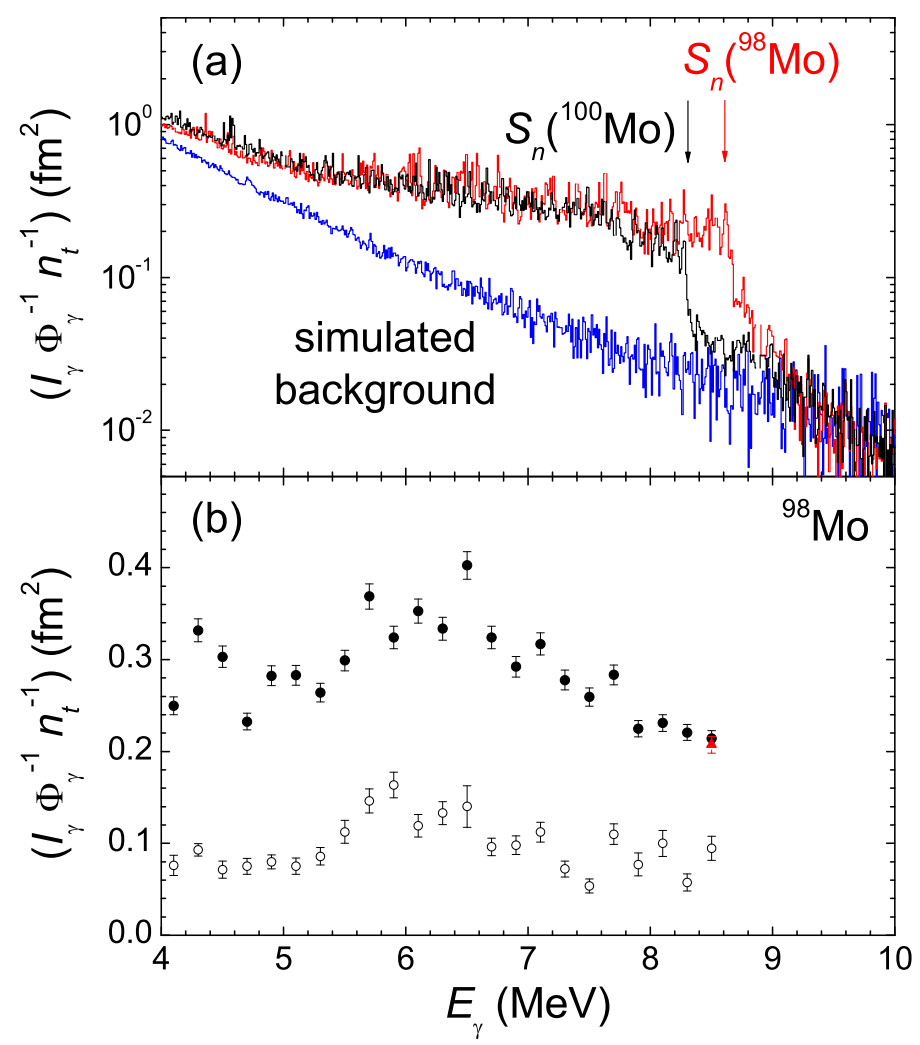

Figure 3: Comparison of the experimental spectra (a) from ${ }^{98} \mathrm{Mo}$ (red) and ${ }^{100} \mathrm{Mo}$ (black) measured at $E_{e}^{\mathrm{kin}}=$ 13.2 MeV corrected for the full-energy peak efficiency, the photon flux, the area density of the target atoms and the measuring time, with simulations for the non-resonant background (blue). The intensities of the peaks from ${ }^{11} \mathrm{~B}$ and neutron-capture induced background are subtracted from the experimental spectra. (b) Dipole strength in ${ }^{98} \mathrm{Mo}$ deduced from the resolved peaks (open circles), from subtraction of the background taken from the ${ }^{100} \mathrm{Mo}$ spectrum (red triangle at $8.5 \mathrm{MeV}$ ) and from the quasi-continuum (filled circles) after subtraction of the simulated background.

density of the target atoms and the measuring time. The intensities of the peaks from ${ }^{11} \mathrm{~B}$ are subtracted from the experimental spectra. The two spectra are characterised by a sudden decrease of observed intensity beyond the neutron-separation energy caused by the dominant emission of neutrons. Therefore, above $S_{\mathrm{n}}$ the spectrum contains nearly only non-resonant background, i.e. counts in the spectrum which do not result from deexcitations of nuclear levels. Note that the non-resonant background depends on the amount of the target material. Therefore the experiments on ${ }^{98} \mathrm{Mo}$ and ${ }^{100} \mathrm{Mo}$ were carried out for almost identical targets with a difference in mass of only about $1 \%$. According to the different neutron-separation energies in ${ }^{98} \mathrm{Mo}\left(S_{n}=8.64 \mathrm{MeV}\right)$ and ${ }^{100} \mathrm{Mo}\left(S_{n}=8.29 \mathrm{MeV}\right)$ one finds a range between 8.3 and $8.6 \mathrm{MeV}$ in the spectrum of ${ }^{98} \mathrm{Mo}$ for which the non-resonant background can be taken from the spectrum of ${ }^{100} \mathrm{Mo}$. The residuum above the spectrum of ${ }^{100} \mathrm{Mo}$ represents the intensity of the resonant $\gamma$-rays in ${ }^{98} \mathrm{Mo}$. The intensity in the considered energy range is compared with the intensity deduced from the identified peaks in Fig. 3 (b). The ratio of the strength deduced from the experimental spectrum after subtraction of the nonresonant background to the strength of the resolved peaks at $E_{\gamma}=8.5 \mathrm{MeV}$ is about three which 


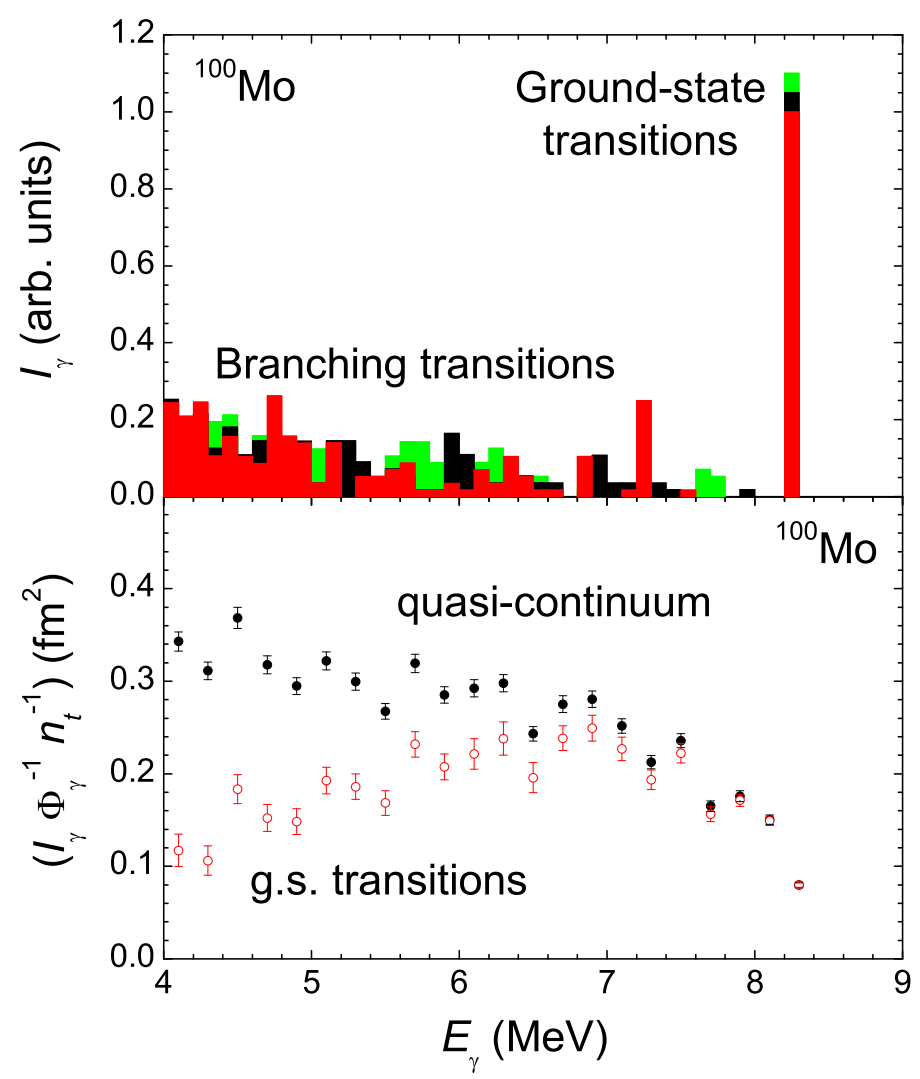

Figure 4: Top: Simulated spectrum of $\gamma$-rays deexciting levels in ${ }^{100} \mathrm{Mo}$ in the bin from 8.2 to $8.3 \mathrm{MeV}$. Bottom: Intensity of the quasi-continuum in ${ }^{100} \mathrm{Mo}$ (filled circles) and the remaining intensity distribution of ground-state transitions after removing the branching intensities (red open circles).

shows that only $30 \%$ of the strength is located in resolved peaks.

It is important to extend the estimate of the non-resonant background in the measured spectrum to lower energies in order to determine the total strength in the nucleus. For that purpose we performed GEANT3 simulations of the non-nuclear processes in the target leading to background in the measured spectra.

The simulated non-resonant background is compared with the spectra of ${ }^{98} \mathrm{Mo}$ and ${ }^{100} \mathrm{Mo}$ in Fig. 3 (a). The distribution of the intensity from ${ }^{98} \mathrm{Mo}$ and ${ }^{100} \mathrm{Mo}$ is obtained by subtraction of the simulated non-resonant background from the experimental spectra. This distribution forms a quasicontinuum and contains the intensity of the identified peaks and the continuous intensity as a result of overlapping weak deexcitations. The apparent strength in the quasi-continuum is compared with the strength from the identified peaks in Fig. 3 (b). The comparison shows that in the range from 4 $\mathrm{MeV}$ to $S_{n}$ only $20 \%$ to $50 \%$ of the strength is located in the peaks.

\subsection{Dipole strength deduced from a quasi-continuous spectrum}

The quasi-continuum resulting from the subtraction of the non-resonant background from the experimental spectrum contains the ground-state transitions (elastic scattering) and in addition, transitions to lower-lying excited states (branching transitions) as well as transitions from such 


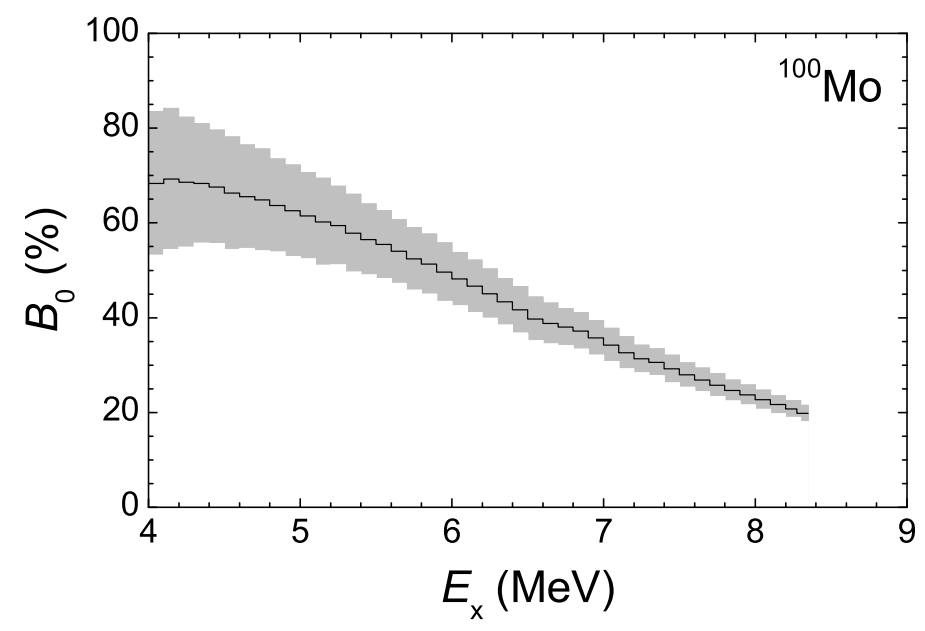

Figure 5: Distribution of the mean branching ratios $B_{0}$ deduced from the cascade simulations for ${ }^{100} \mathrm{Mo}$ over 1000 nuclear realisations. Hatched area shows the $1 \sigma$-variation from the mean value due to fluctuations of the level spacings and widths.

states to the ground state (cascade transitions) which cannot be distinguished in our experiments. The first step of the analysis of the quasi-continuum is an estimate of branching and cascade transitions. Cascade simulations were performed by the Monte-Carlo procedure as described above. Spectra of $\gamma$-ray cascades were binned into intervals with a width of $\Delta=100 \mathrm{keV}$. Examples of spectra deexciting levels from $E_{x}=8.2$ to $8.3 \mathrm{MeV}$ in ${ }^{100} \mathrm{Mo}$ from three nuclear realisations are given in the top part of Fig. 4. Starting from the high-energy end of an experimental spectrum, which contains ground-state transitions only, the simulated intensities of the ground-state transitions were normalised to the experimental ones in the considered bin and the intensity distribution of the branching transitions is subtracted from the experimental spectrum. Applying this procedure step-by-step for each energy bin moving towards the low-energy end of the spectrum one obtains an intensity distribution that contains ground-state transitions only. The correction for the branching and cascade transitions is performed for every nuclear realisation and a mean spectrum of the ground-state transitions is calculated. This mean intensity distribution, which is related to the elastic-scattering cross section $\sigma_{\gamma \gamma}$, is compared with the uncorrected quasi-continuum in the lower part of Fig. 4.

The distribution of the branching ratios $B_{0}$ for ${ }^{100} \mathrm{Mo}$ is simultaneously deduced from the simulations of the $\gamma$-ray cascades is shown in Fig. 5. The branching ratios $B_{0}$ are calculated as ratio of the summed intensity $\sum_{\Delta} I_{\gamma}^{\text {g.s. }}$ of the ground-state transitions of the levels in a $\Delta=100 \mathrm{keV}$ bin, to the summed intensity $\sum_{\Delta} I_{\gamma}^{\text {all }}$ of all transitions deexciting these levels. According to Eqs. (2.1) and (2.2) we obtain the relation:

$$
B_{0}=\frac{\sum_{\Delta} I_{\gamma}^{\mathrm{g} . \mathrm{s}}}{\sum_{\Delta} I_{\gamma}^{\text {all }}}=\frac{\sum_{i \text { in } \Delta} \Gamma_{0}^{i} B_{0}^{i}}{\sum_{i \text { in } \Delta} \Gamma_{0}^{i}} .
$$

It can be seen that the values of $B_{0}$ for ${ }^{100} \mathrm{Mo}$ are some $60 \%$ at low excitation energy where the levels do not have many possibilities to deexcite to even lower-lying levels. Towards high energy, however, the values of $B_{0}$ drop to about $20 \%$, which shows that high-lying levels deexcite via many 


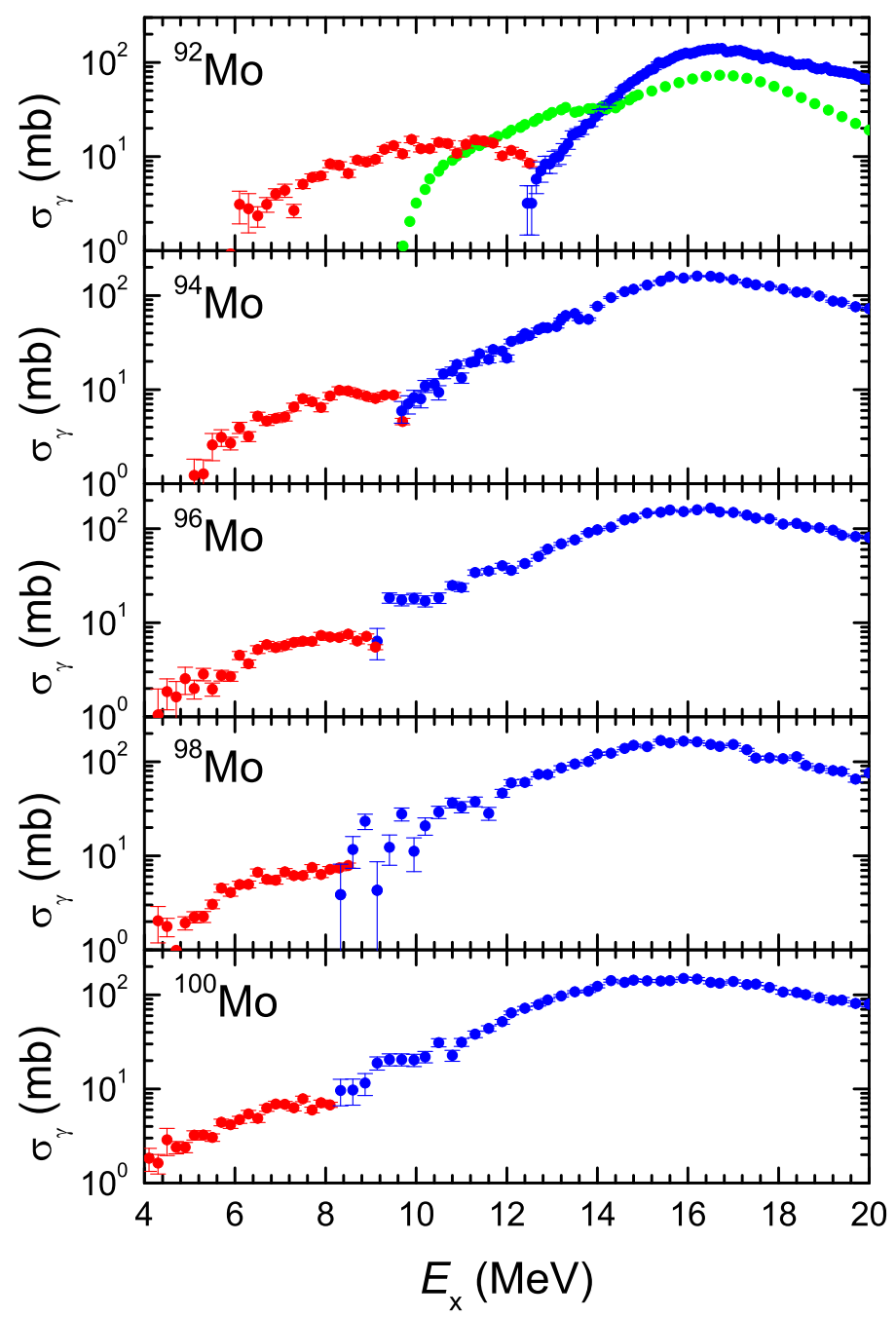

Figure 6: Comparison of the absorption cross section determined from our photon-scattering experiments (red circles) and from measurements of the $(\gamma, n)$ reaction [32] (blue circles). The $(\gamma, p)$ cross sections for ${ }^{92}$ Mo (green circles) are calculated with the code "Talys" [34].

different branches to lower-lying states. Dividing the intensities of the ground-state transitions, which are proportional to the elastic scattering cross sections $\sigma_{\gamma \gamma}$, by the corresponding branching ratios $B_{0}$ (see Fig. 5), we obtain the absorption cross sections $\sigma_{\gamma}=\sigma_{\gamma \gamma} / B_{0}$.

The photoabsorption cross sections $\sigma_{\gamma}$ determined in this way are presented in Fig. 6 . They are compared with $(\gamma, n)$ cross sections [32]. The $(\gamma, n)$ data were scaled with a factor of 0.86 according to the findings in Ref. [33]. In order to show the effect of the reconstruction of the dipole-strength distributions we compare the results for $\sigma_{\gamma}$ with the still uncorrected quasi-continuum. The deduced $\sigma_{\gamma}$ are compared with results from ${ }^{98} \operatorname{Mo}(n, \gamma){ }^{99}$ Mo experiments [6] applying Eq. (2.8).

\section{Discussion}

The method of analysis of the photon-scattering experiments allowed us determination of the 

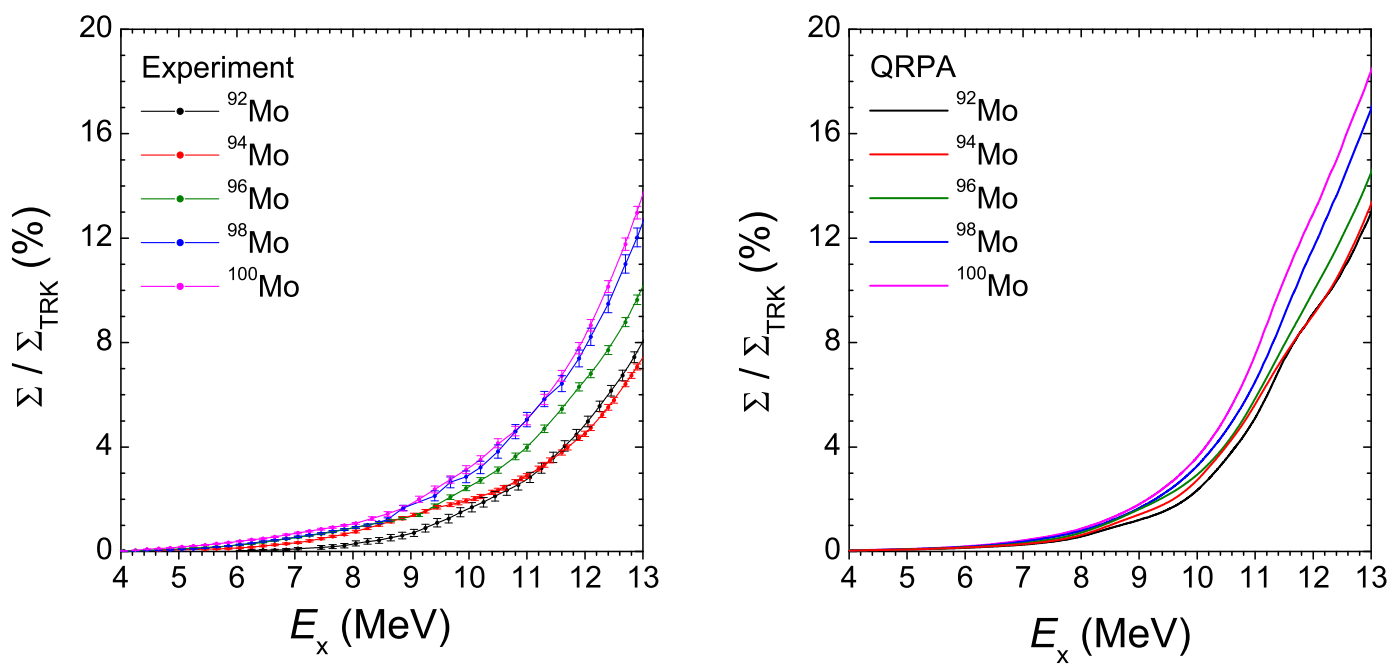

Figure 7: Plot of the cumulative integrated photoabsorption cross section for the stable even-mass Mo isotopes obtained from the photon-scattering experiments and the $(\gamma, \mathrm{n})$ measurements [32]. The same plot compared with predictions from the QRPA (right panel). The calculated strength is smeared with Lorentzian function with a width of $0.5 \mathrm{MeV}$.

photoabsorption cross section below the neutron separation energy. The dipole-strength distributions in the stable even-mass Mo isotopes were obtained combining the results from the $\left(\gamma, \gamma^{\prime}\right)$ and $(\gamma, \mathrm{n})[32]$ experiments (cf. Fig. 6). In order to examine the behaviour of the dipole-strength distributions we apply QRPA calculations in a deformed basis [35]. The QRPA calculations for the $1^{-}$ excitations use the following Hamiltonian:

$$
H_{E 1}^{\mathrm{QRPA}}=h_{\mathrm{MF}}-\frac{1}{2} \sum_{\mu=-1,+1}^{\mathrm{t}=0,1} \kappa_{1 \mu}^{\mathrm{t}} Q_{1 \mu}^{\mathrm{t}} Q_{1-\mu}^{\mathrm{t}}-\frac{1}{2} \sum_{\mu=-3,+3}^{\mathrm{t}=0,1} \kappa_{3 \mu}^{\mathrm{t}} Q_{3 \mu}^{\mathrm{t}} Q_{3-\mu}^{\mathrm{t}} .
$$

The term $h_{\mathrm{MF}}$ in Eq. (4.1) includes the Nilsson mean field plus monopole pairing using the equilibrium deformation, see Ref.[21]. The following terms denote the isoscalar $(t=0)$ and isovector $(t=1)$ part of the dipole-dipole interaction $(\lambda=1)$ and octupole-octupole interaction $(\lambda=3)$ defining the multipole operators by $Q_{\lambda \mu}^{\mathrm{t}}=\left[r^{\lambda} Y_{\lambda \mu}\right]^{\pi}+(-)^{\mathrm{t}}\left[r^{\lambda} Y_{\lambda \mu}\right]^{v}$. The $E 1$ transition operator is given by:

$$
\widehat{\mathbf{E 1}}=e\left[r Y_{1 \mu}\right]^{\pi} .
$$

The excitation energies and the $E 1$ transition strengths of $J^{\pi}=1^{-}$states were calculated using the QRPA with doubly stretched coordinates [36]. It is important to take the deformation degree of freedom in the QRPA calculations into account because the nuclear shape changes from the spherical isotope ${ }^{92} \mathrm{Mo}_{50}$ to a triaxial shape in the isotopes ${ }^{98} \mathrm{Mo}_{56}$ and ${ }^{100} \mathrm{Mo}_{58}$ [37]. The isoscalar constants $\kappa_{\lambda \mu}^{\mathrm{t}=1}$ were chosen to be large enough to eliminate the spurious centre of mass motion according to the suppression method [38]. For calculating the $E 1$ strength the only strength parameter which is not determined by the selfconsistency condition is the relative strength of the isovector interaction term. This parameter has been adapted to the position of the maximum of the GDR. In order to examine the behaviour of the dipole-strength distributions we calculated the cumulative integrated cross section $\Sigma=\sum_{0}^{E_{\mathrm{x}}} \sigma_{\gamma}(E) \Delta E$ with the QRPA. In Fig. 7 the cumulative integrated cross 

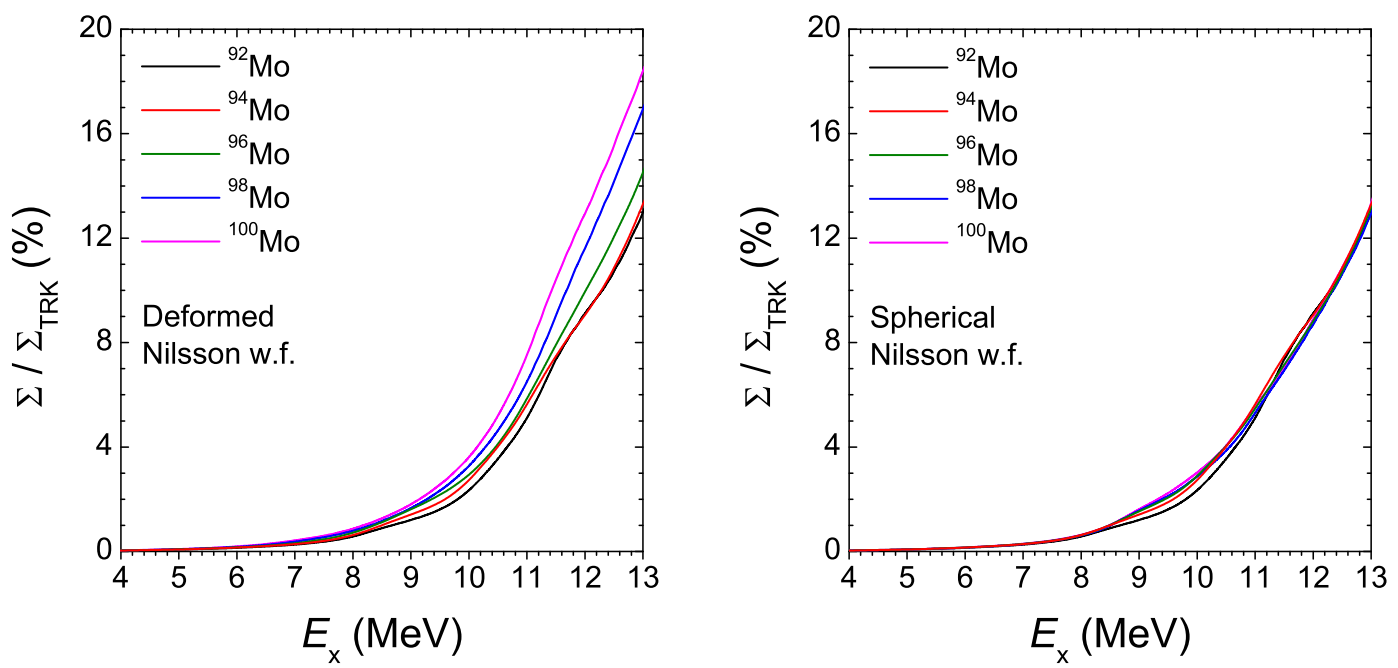

Figure 8: Comparison of the calculated cumulative integrated cross sections for ${ }^{92} \mathrm{Mo},{ }^{98} \mathrm{Mo}$ and ${ }^{100} \mathrm{Mo}$ for the case of a) deformed Nilsson wave functions and b) spherical Nilsson wave functions. The calculated strength is smeared with Lorentzian function with a width of $0.5 \mathrm{MeV}$.

sections normalised to the values given by the Thomas-Reiche-Kuhn sum rule $\Sigma_{\mathrm{TRK}}=60 \mathrm{NZ} / \mathrm{Amb}$ $\mathrm{MeV}$ are displayed for the studied isotopes and compared with the experimental data. One sees that $\Sigma$ increases from ${ }^{92} \mathrm{Mo}$ to ${ }^{100} \mathrm{Mo}$ by about a factor of two at $8 \mathrm{MeV}$, close to the neutron threshold of ${ }^{100} \mathrm{Mo}$.

The QRPA calculations reproduce in particular the relative increase of the $\Sigma$ with increasing $N / Z$. An increase of the dipole strength with the neutron number $N$ is usually attributed to the so called Pygmy Dipole Resonance (PDR), a concentration of electric-dipole (E1) strength in the energy range between 6 and $10 \mathrm{MeV}$, which is considered as an out-of-phase oscillation of a neutron skin against an inert core. The properties of the PDR are therefore expected to depend strongly on the ratio of neutron number to proton number $N / Z$. The distributions in Fig. 7 have a smooth energy dependence and do not indicate a strong PDR structure. The enlargement of $\Sigma$ with increasing $N / Z$ seems to be related to the influence of the increasing deformation. This has been proven by performing QRPA calculations with a spherical shape for ${ }^{96} \mathrm{Mo},{ }^{98} \mathrm{Mo}$ and ${ }^{100} \mathrm{Mo}$, shown in Fig. 8 , that predict $\Sigma$ resg. 8. In fact, the effect of the deformation as the reason of an enlargement of the photoabsorption cross section is plausible. It is well known that the width of GDR gets considerably broadened by deformation [39] which leads to a lift of strength in the tail because the total value of the photoabsorption cross section is limited by the energy-weighted sum rule.

\section{Summary}

The response of the stable even-mass molybdenum isotopes to dipole radiation was investigated at the bremsstrahlung facility at the superconducting linear electron accelerator ELBE of the Forschungszentrum Dresden-Rossendorf. The photon-scattering experiments were carried out at an electron-beam energy higher than the neutron-separation energy of the Mo isotopes which allowed us to study the dipole strength close to the threshold for the $(\gamma, n)$ reaction. 
Because of the Porter-Thomas fluctuations of the level widths most of the levels do not appear as prominent peaks in the experimental spectra. Due to the high level density above $5 \mathrm{MeV}$ and the finite resolution of the detectors weakly populated levels produce a quasi-continuum of overlapping peaks. It is shown from a comparison of the spectra of ${ }^{98} \mathrm{Mo}$ and ${ }^{100} \mathrm{Mo}$ that close to the neutronseparation energy of ${ }^{98} \mathrm{Mo}$ the strength in the quasi-continuum is two times higher than the strength in the peaks. GEANT3 simulations were applied to determine the non-resonant background in the measured spectra and thus to extract the quasi-continuum. The continuum contains the groundstate transitions as well as the branching transitions to the low-lying levels and the subsequent deexcitations of these levels.

A Monte Carlo code for simulations of $\gamma$-ray cascade was developed which allows us (i) to deconvolute the intensity distribution of the ground-state transitions from the quasi-continuum and thus to determine the cross section for elastic scattering and (ii) to calculate the branching ratios for deexcitations to the ground state and to obtain the photoabsorption cross section. Since the obtained photoabsorption cross sections for the molybdenum isotopes match the measured cross sections for the $(\gamma, n)$ reaction the method allows us to determine the tail of the Giant Dipole Resonance below the neutron-separation energy. In order to test the results of the simulations experimentally, in particular the calculated branching ratios, we plan an experiment with a monoenergetic photon beam at the HI $\gamma \mathrm{S}$ facility of the Duke University.

The obtained dipole-strength distributions for the stable even-mass Mo isotopes show that the strength increases with the neutron number. The experimental results are discussed in the frame of QRPA calculations in a deformed basis which describe the increasing strength as a result of the increasing deformation.

This work was supported by the Deutsche Forschungsgemeinschaft under contract DO-466/1-2.

\section{References}

[1] M. Goldhaber and E. Teller, Phys. Rev. 74, 1046 (1948).

[2] H. Steinwedel and J. Jensen, Z. Naturforschung 5A, 413 (1950).

[3] W. D. Myers, et al., Phys. Rev. C 15, 2032 (1977).

[4] C. B. Dover, et al., Ann. Phys. (N.Y.) 70, 458 (1972).

[5] J. Wambach, Rep. Prog. Phys. 51, 989 (1988).

[6] J. Kopecky and M. Uhl, Proceedings of the NEA/ENEA and IAEA Specialists' Meeting on Measurement, Calculation and Evaluation of Photon Production Data, Bologna, November 9-17, 1994.

[7] S. G. Kadmenskij, et al., Yad. Fiz. 37, 277 (1983).

[8] P. Axel, Phys. Rev. C 126, 671 (1962).

[9] R. M. Laszewski and P. Axel, Phys. Rev. C 19, 342 (1979).

[10] R. Alarcon, et al., Phys. Rev. C 36, 954 (1987).

[11] G. A. Bartholomew, et al., Adv. Nucl. Phys. 7, 229 (1972).

[12] N. Ryezayeva, et al., Phys. Rev. Lett. 89, 272502 (2002). 
[13] B. L. Berman and S. C. Fultz, Rev. Mod. Phys. 47, 713 (1975).

[14] S. Goko, et al., Phys. Rev. Lett. 96, 192501 (2006).

[15] F. R. Metzger, Prog. Nucl. Phys. 7, 54 (1959).

[16] U. Kneissl, H. H. Pitz, and A. Zilges, Prog. Part. Nucl. Phys. 37, 349 (1996).

[17] F. Běcvář, Nucl. Instrum. Methods Phys. Res. Sec. A 417, 434 (1998).

[18] T. von Egidy and D. Bucurescu, Phys. Rev. C 72, 044311 (2005).

[19] Y. Kalmykov, et al., Phys. Rev. Lett. 96, 012502 (2006).

[20] T. A. Brody, et al., Rev. Mod. Phys. 53, 385 (1981).

[21] G. Rusev, R. et al., Phys. Rev. C 73, 044308 (2006).

[22] F. Běcvár̆, et al., Phys. Rev. C 52, 1278 (1995).

[23] M. Krtička, et al., Phys. Rev. Lett. 92, 172501 (2004).

[24] http://www-nds.iaea.org/RIPL-2/.

[25] C. E. Porter and R. G. Thomas, Phys. Rev. 104, 483 (1956).

[26] R. Schwengner, et al., Nucl. Instrum. Methods Phys. Res. Sec. A 555, 211 (2005).

[27] A. Wagner, et al., J. Phys. (London) G31, S1969 (2005).

[28] G. Roche, C. Ducos, and J. Proriol, Phys. Rev. A 5, 2403 (1972).

[29] E. Haug, private communications.

[30] CERN Program Library Long Writeup W5013, Geneva 1993, unpublished.

[31] R. Schwengner, et al., contribution to these proceedings.

[32] H. Beil, et al., Nucl. Phys. A227, 427 (1974).

[33] B. L. Berman, et al., Phys. Rev. C 36, 1286 (1987).

[34] A. Koning, et al., AIP Conf. Proc. 769, 177 (2005).

[35] F. Dönau, et al., Phys. Rev. C 76, 014317 (2007).

[36] H. Sakamoto and T. Kishimoto, Nucl. Phys. A501, 205 (1989).

[37] P. Möller and J. R. Nix, At. Data Nucl. Data Tables 26, 165 (1981).

[38] F. Dönau, Phys. Rev. Lett. 94, 092503 (2005).

[39] A. Bohr and B. R. Mottelson, Nuclear Structure, W. A. Benjamin, Massachusetts (1975). 